\title{
The GBT350 Survey of the Northern Galactic Plane for Radio Pulsars and Transients
}

\author{
J. W. T. Hessels*, S. M. Ransom ${ }^{\dagger}$, V. M. Kaspi**, M. S. E. Roberts, D. J. \\ Champion** and B. W. Stappers $^{\S}$ \\ *Astronomical Institute “Anton Pannekoek”, University of Amsterdam, Kruislaan 403, 1098 SJ Amsterdam, The \\ Netherlands \\ ${ }^{\dagger}$ National Radio Astronomy Observatory, 520 Edgemont Road, Charlottesville, VA 22903, U.S.A. \\ ${ }^{* *}$ Department of Physics, McGill University, Montreal, QC H3A 2T8, Canada \\ ${ }^{\ddagger}$ Eureka Scientific, Inc., Oakland, CA 94602-3017, U.S.A. \\ $\S$ Jodrell Bank Centre for Astrophysics, University of Manchester, Manchester, M13 9PL, UK
}

\begin{abstract}
.
Using the Green Bank Telescope (GBT) and Pulsar Spigot at $350 \mathrm{MHz}$, we have surveyed the Northern Galactic Plane for pulsars and radio transients. This survey covers roughly 1000 square degrees of sky within $75^{\circ}<l<165^{\circ}$ and $|b|<5.5^{\circ}$, a region of the Galactic Plane inaccessible to both the Parkes and Arecibo multibeam surveys. The large gain of the GBT along with the high time and frequency resolution provided by the Spigot make this survey more sensitive by factors of about 4 to slow pulsars and more than 10 to millisecond pulsars (MSPs), compared with previous surveys of this area. In a preliminary, reduced-resolution search of all the survey data, we have discovered 33 new pulsars, almost doubling the number of known pulsars in this part of the Galaxy. While most of these sources were discovered by normal periodicity searches, 5 of these sources were first identified through single, dispersed bursts. We discuss the interesting properties of some of these new sources. Data processing using the data's full-resolution is ongoing, with the goal of uncovering MSPs missed by our first, coarse round of processing.
\end{abstract}

Keywords: Pulsar; Survey; Radio; RRAT; GBT

PACS: $97.60 . \mathrm{Gb}$

\section{INTRODUCTION}

Here we define the "Northern Sky" as the sky above the northern Arecibo declination limit of $38^{\circ}$. Previous pulsar surveys of the Northern Sky between $300-500 \mathrm{MHz}$ include an all-sky survey above $\delta>38^{\circ}$ using the Jodrell Bank telescope at $428 \mathrm{MHz}$, which, largely due to RFI, discovered only one pulsar [1]; a survey at Green Bank using the $140-\mathrm{ft}(43-\mathrm{m})$ equatorially mounted telescope at $370 \mathrm{MHz}$, which found eight new pulsars [2]; and a survey using the Green Bank 300-ft (91-m) transit telescope at $390 \mathrm{MHz}$, which provided partial coverage of the Northern Sky and discovered 20 pulsars [3].

The GBT's large gain $(2 \mathrm{~K} / \mathrm{Jy})$ and maneuverability make it the most sensitive radio telescope on Earth over a large portion of the sky. Furthermore, the primary beam width of the GBT at $350 \mathrm{MHz}$ is $\sim 0.6^{\circ}$, large enough to permit very efficient large-scale, single-pixel surveys of the sky. This motivated us to commence a $350-\mathrm{MHz}$ survey, which we will refer to as the GBT350 survey, of the Northern Galactic Plane, corresponding to Galactic longitudes $75^{\circ}<l<165^{\circ}$ and out to latitudes $|b|<5.5^{\circ}$. Here we present a short summary of the survey design and analysis techniques, and then discuss some of the new sources.

\section{OBSERVATIONS \& ANALYSIS}

Data collection began in April 2005 and is now mostly complete. A total of $\sim 4000$ pointings were required to cover the survey area, which is shown in Figure 1] Data were recorded at a centre frequency of $350 \mathrm{MHz}$ using the GBT Pulsar Spigot [4] in a $50 \mathrm{MHz}$ bandwidth mode with 1024 lags and $81.92 \mu$ s sampling. With this spectral resolution, there is a residual dispersive smearing of $100(1000) \mu \mathrm{s}$ at $\mathrm{DM}=10(100) \mathrm{pc} \mathrm{cm}^{-3}$. Thus, these data provide good sensitivity to even the fastest known MSPs, up to a DM of roughly $100 \mathrm{pc} \mathrm{cm}^{-3}$. The integration time per pointing is $120 \mathrm{~s}$, resulting in a survey sensitivity to normal, slow pulsars of roughly $S_{400}^{\min }=2 \mathrm{mJy}$. The GBT350 survey is a factor of $>4$ times more sensitive to slow pulsars and $>10$ times more sensitive to fast pulsars at moderate DMs than previous surveys of the same area. Furthermore, the current RFI environment at Green Bank in this frequency range is remarkably good. This allows us to use a very low signal-to-noise threshold when investigating candidates.

We have analyzed all the survey pointings at reduced resolution in order to first pick out the slower $\left(P_{\text {spin }}>\right.$ $50 \mathrm{~ms}$ ) pulsars. This processing takes only $10 \%$ the time of full resolution processing and is capable of finding 


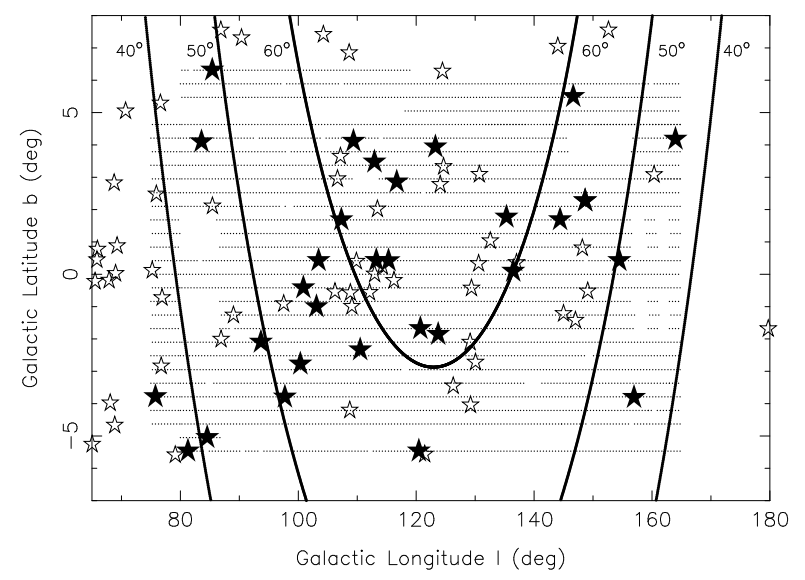

FIGURE 1. The GBT350 survey area shown in Galactic coordinates. Observed pointing positions are marked with dots. Empty and filled stars denote the positions of known and newly found pulsars respectively. Constant lines of declination at $40^{\circ}$, $50^{\circ}$, and $60^{\circ}$ are also shown.

the vast majority of the potentially detectable sources. We are currently re-processing the data at full resolution and expect that this will reveal a few more sources, such as MSPs, whose properties make them undetectable at lower resolution. We have employed both standard periodicity searches, summing up to 16 harmonics and including a narrow search in acceleration space, as well as single pulse searches for bright, dispersed bursts. We are also considering searching the data for very slow $\left(P_{\text {spin }}>4 \mathrm{~s}\right)$ pulsars with a fast-folding algorithm, as well as re-searching the data using a larger number of summed harmonics to increase sensitivity to very short-duty cycle $(<2 \%)$ pulsars.

\section{RESULTS}

Thus far, we have discovered 33 pulsars, of which 5 were discovered through their bright pulses in our single pulse search. There are 39 previously known pulsars in this part of the Galaxy and thus the GBT350 survey has significantly increased the known pulsar population in the Nothern Galactic Plane. These new sources and their basic properties are summarized in Table 1 . We are timing all these new pulsars with GBT and Westerbork and have timing solutions for 12 so far. We now briefly discuss the interesting properties of some of the new systems:

PSR J0426+4933: Westerbork observations of this pulsar at $1.4 \mathrm{GHz}$ have revealed two distinct components in its cumulative pulse profile. Preliminary analysis indicates that the second, brighter, and extremely narrow component is composed of individual bright pulses. The duty cycle of these individual pulses is only $\sim$
TABLE 1. New Pulsars from the GBT350 Survey. We are timing all of these sources, and those marked with an asterisk already have precise astrometric and spin parameters. The source PSR J2029+45 was also discovered with Westerbork (Stappers \& Janssen, private communication) and the sources PSRs J2208+5500 and J2217+5733 with GMRT (McLaughlin, private communication, also in this volume).

\begin{tabular}{|c|c|c|c|}
\hline Pulsar & $\begin{array}{c}\text { Period } \\
(\mathrm{ms})\end{array}$ & $\begin{array}{c}\text { DM } \\
\left(\mathrm{pc} \mathrm{cm}^{-3}\right)\end{array}$ & Notes \\
\hline $\mathrm{J} 0033+57$ & 315 & 76 & \\
\hline $\mathrm{J} 0033+61$ & 912 & 37 & \\
\hline J0054+66 & 1390 & 15 & Nearby, RRAT \\
\hline $\mathrm{J} 0058+6125^{*}$ & 637 & 129 & Extreme Nuller \\
\hline $\mathrm{J} 0240+62$ & 592 & 4 & Nearby, Low-lum \\
\hline $\mathrm{J} 0243+6027^{*}$ & 1473 & 141 & \\
\hline $\mathrm{J} 0341+5711^{*}$ & 1888 & 100 & \\
\hline $\mathrm{J} 0408+55 \mathrm{~A}$ & 1837 & 55 & \\
\hline $\mathrm{J} 0408+55 \mathrm{~B}$ & 754 & 64 & \\
\hline $\mathrm{J} 0413+58$ & 687 & 57 & \\
\hline $\mathrm{J} 0419+44$ & 1241 & 71 & \\
\hline J0426+4933* & 922 & 85 & \\
\hline $\mathrm{J} 0519+44$ & 515 & 52 & \\
\hline $\mathrm{J} 2024+48$ & 1262 & 99 & \\
\hline $\mathrm{J} 2029+45$ & 1099 & 228 & \\
\hline $\mathrm{J} 2030+55$ & 579 & 60 & \\
\hline $\mathrm{J} 2038+35$ & 160 & 58 & \\
\hline $\mathrm{J} 2043+7045^{*}$ & 588 & 57 & \\
\hline $\mathrm{J} 2102+38$ & 1190 & 85 & \\
\hline $\mathrm{J} 2111+40$ & 4061 & 120 & Slow rotator \\
\hline $\mathrm{J} 2138+4911^{*}$ & 696 & 168 & \\
\hline $\mathrm{J} 2203+50$ & 745 & 79 & \\
\hline $\mathrm{J} 2208+5500^{*}$ & 933 & 105 & \\
\hline $\mathrm{J} 2213+53$ & 751 & 161 & \\
\hline $\mathrm{J} 2217+5733^{*}$ & 1057 & 130 & \\
\hline $\mathrm{J} 2222+5602^{*}$ & 1336 & 168 & Periodic Nuller \\
\hline $\mathrm{J} 2238+6021^{*}$ & 3070 & 182 & Slow rotator \\
\hline $\mathrm{J} 2244+63$ & 461 & 92 & \\
\hline $\mathrm{J} 2315+58$ & 1061 & 74 & \\
\hline $\mathrm{J} 2316+64$ & 216 & 248 & \\
\hline $\mathrm{J} 2326+6141^{*}$ & 790 & 33 & \\
\hline $\mathrm{J} 2343+6221^{*}$ & 1799 & 117 & \\
\hline $\mathrm{J} 2352+65$ & 1164 & 152 & \\
\hline
\end{tabular}

$0.2 \%$, roughly $2 \mathrm{~ms}$. Deeper studies into the pulse energy distribution and single pulse characteristic of PSR J0426+4933 are planned. Are these pulses "giant pulse like", or is PSR J0426+4933, like PSR B0656+14 [5], a pulsar that falls in between the groups of pulsars with "giant pulses", 'giant micropulses" [6], and the RRATs [7]?

PSR J2222+5601: This pulsar shows what looks like quasi-periodic nulling, with roughly constant emission for $\sim 120 \mathrm{~s}$ followed by $\sim 30 \mathrm{~s}$ gaps. We are using the GBT to investigate how strictly periodic these nulls are. A few other examples of pulsars showing periodic nulls have recently been identified (e.g. PSR B1133+16, Herfindal et al. in this volume). This emission behaviour is also reminiscent of that recently identified in the 
so-called 'sometimes a pulsar" PSR B1931+24 [8], although the behaviour seen here is on a much shorter timescale.

PSR J0058+6125: This pulsar is nulled during approximately $97 \%$ of its pulse periods, placing it closer to the RRATs [7] than the normal pulsar population in terms of its visibility. This is a considerably larger nulling fraction than those typically observed in nulling pulsars [consider for instance 9]. Because of the difficulty in discovering such an intermittent pulsar, many similar systems are likely to exist, but have gone undetected. Nulling of PSR J0058+6125 has been observed to last for up to about $20 \mathrm{~min}$ (close to 2000 pulse periods) and, intriguingly, the emission appears to come in windows of $100-200 \mathrm{~s}$, in which numerous, closely spaced pulses are seen. Nulling also occurs within windows where the pulsar is visible. Thus, it appears that this pulsar has two nulling timescales: one on the order of the pulse period, the other on the order of several minutes.

PSR J0240+62: This source, identified by its bright pulses in our single pulse search, has a DM of only $\sim 4 \mathrm{pc}$ $\mathrm{cm}^{-3}$. This is extremely low (only 6 of the $\sim 1800$ known pulsars have a lower DM), and suggests that this pulsar is very nearby. Using the NE2001 electron model of the Galaxy [10], the inferred distance is a mere $400 \mathrm{pc}$, making it one of the closest known pulsars to Earth. Followup observations of this source are needed to determine its radio flux. If the source is indeed as weak as it appears to be in its discovery observation, then its proximity also indicates that it is a very low-luminosity source. Such sources are important for mapping the low-luminosity end of the pulsar luminosity distribution and the discovery of this source bodes well for the discovery of more such sources with LOFAR [see 11, for a description of possible pulsar-related LOFAR science]. Due to its proximity, we are also planning X-ray observations of this source.

PSR J0054+66: This source was very recently discovered via its occasional strong, dispersed bursts, one of which is shown in Figure 2 Though we have currently only observed 8 bursts from this source, it appears that they are spaced in multiples of $1.3903 \mathrm{~s}$ and occur once every $\sim 100-1000 \mathrm{~s}$. This implies that PSR J0054+66 is an RRAT-like source, showing very sporadic radio pulsations. It has a low DM of $14.5 \mathrm{pc} \mathrm{cm}^{-3}$ and a corresponding DM distance of only $1 \mathrm{kpc}$. PSR J0054+66's proximity bodes well for future studies in X-rays. What is needed to better study this source in radio is longer continuous observations to better characterize the distribution of the bursts in intensity and time. This will then determine how this intriguing new source fits in with the known population of RRATs.

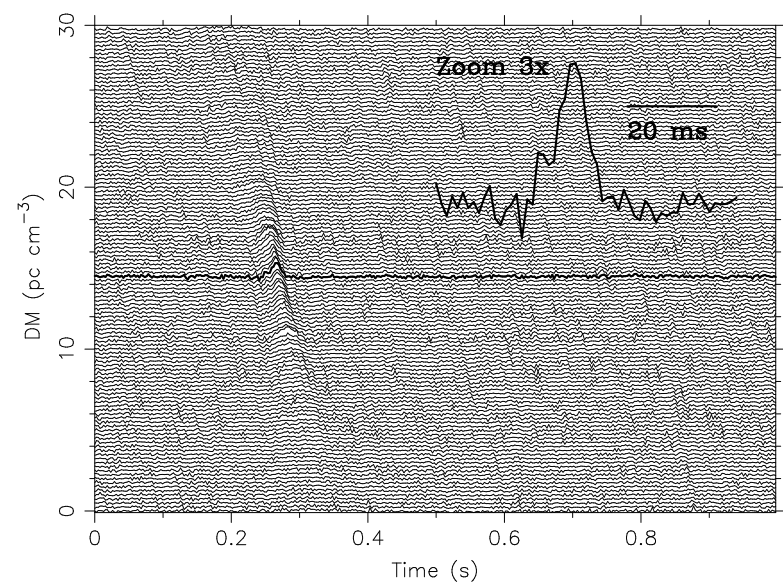

FIGURE 2. Main Panel: A 1-s section of the discovery observation of PSR J0054+66. A bright burst is shown as a function of DM and time. The signal peaks at $14.5 \mathrm{pc} \mathrm{cm}^{-3}$ and is smeared at DMs above and below this. Inset: Zoom-in of the same burst, properly dedispersed. The burst has a least two components.

\section{ACKNOWLEDGMENTS}

J.W.T.H. thanks NSERC and the Canadian Space Agenncy for a postdoctoral fellowship and supplement respectively.

\section{REFERENCES}

1. L. Nicastro, A. G. Lyne, D. R. Lorimer, P. A. Harrison, M. Bailes, and B. D. Skidmore, MNRAS 273, L68 (1995).

2. R. W. Sayer, D. J. Nice, and J. H. Taylor, ApJ 474, 426 (1997).

3. G. H. Stokes, D. J. Segelstein, J. H. Taylor, and R. J. Dewey, ApJ 311, 694-700 (1986).

4. D. L. Kaplan, R. P. Escoffier, R. J. Lacasse, K. O’Neil, J. M. Ford, S. M. Ransom, S. B. Anderson, J. M. Cordes, T. J. W. Lazio, and S. R. Kulkarni, PASP 117, 643-653 (2005).

5. P. Weltevrede, G. A. E. Wright, B. W. Stappers, and J. M. Rankin, A\&A 459, 597-597 (2006).

6. S. Johnston, W. van Straten, M. Kramer, and M. Bailes, ApJL 549, L101-L104 (2001).

7. M. A. McLaughlin, A. G. Lyne, D. R. Lorimer, M. Kramer, A. J. Faulkner, R. N. Manchester, J. M. Cordes, F. Camilo, A. Possenti, I. H. Stairs, G. Hobbs, N. D'Amico, M. Burgay, and J. T. O'Brien, Nature 439, 817-820 (2006).

8. M. Kramer, A. G. Lyne, J. T. O'Brien, C. A. Jordan, and D. R. Lorimer, Science 312, 549-551 (2006).

9. N. Wang, R. N. Manchester, and S. Johnston, MNRAS 377, 1383-1392 (2007).

10. J. M. Cordes, and T. J. W. Lazio, astro-ph/0207156 (2002).

11. B. W. Stappers, A. G. J. van Leeuwen, M. Kramer, D. Stinebring, and J. Hessels, astro-ph/0701229 (2007). 